\title{
RNAi of COL1A1 in mesenchymal progenitor cells
}

\author{
Sophia Millington-Ward ${ }^{*}{ }^{1}$, Helena P McMahon ${ }^{1}$, Danny Allen ${ }^{1}$, Gearóid Tuohy ${ }^{1}$, \\ Anna-Sophia Kiang ${ }^{1}$, Arpad Palfi ${ }^{1}$, Paul F Kenna ${ }^{1}$, Peter Humphries ${ }^{1}$ and G Jane Farrar ${ }^{1}$ \\ ${ }^{1}$ Ocular Genetics Unit, Department of Genetics, Trinity College Dublin, Dublin 2, Ireland
}

Given that mutant COL1A1 is known to cause Osteogenesis Imperfecta (OI), tools to modulate COL1A1 expression are likely to be of significant therapeutic value. In this context, we have evaluated RNA interference (RNAi) as a means to downregulate COL1A1 expression in Cos-7 cells and in human mesenchymal progenitor stem cells (MPCs), the latter cells giving rise to bone and therefore representing a target cell type for collagen-related disorders. In addition, allele-specificity, a key factor to the success of RNAi-based suppression, was explored with a view to developing a mutation-independent RNAi-based therapeutic for OI by targeting an intragenic SNP within transcripts derived from the COL1A1 gene. Preferential suppression of individual polymorphic alleles that differed by a single nucleotide was observed.

European Journal of Human Genetics (2004) 12, 864-866. doi:10.1038/sj.ejhg.5201230

Published online 7 July 2004

Keywords: RNAi; polymorphism; COL1A1; gene therapy; stem cell; Osteogenesis Imperfecta

\section{Introduction}

Over 150 COL1A1 mutations give rise to the brittle bone disease Osteogenesis Imperfecta (OI) (http://www. le.ac.uk/ genetics/collagen/). It would therefore be preferable to suppress mutant COL1A1 alleles in a mutation-independent manner. ${ }^{1-4}$ Clearly, efficiency and specificity are key factors in the efficacy of a suppression-based therapeutic and RNA interference (RNAi) represents a powerful new tool. ${ }^{5}$ However, some studies show that RNAi may not be sequence specific at the level of a single nucleotide. ${ }^{5}$ It is therefore imperative, when contemplating using RNAibased therapies, to determine whether allele-specificity really can be achieved.

Mesenchymal progenitor stem cells (MPCs) are stem cells, which can give rise to bone and which represent target cells for OI therapies. Engraftment of donor MPCs has been achieved in children with OI. ${ }^{6}$ However, the use of a patient's own MPCs transduced with a therapeutic gene may aid in the cells' long-term survival. To date, RNAi

*Correspondence: $\operatorname{Dr} \mathrm{S}$ Millington-Ward, Ocular Genetics Unit, Department of Genetics, Trinity College Dublin, Dublin 2, Ireland. Tel: + 3531 6082482; Fax: + 3531 6083848; E-mail: sophia@maths.tcd.ie Received 2 December 2003; revised 16 April 2004; accepted 22 April 2004 has not been shown in MPCs. In this study, short interfering RNAs $\left(\right.$ siRNAs $\left.^{5}\right)$ targeting COL1A1 have been evaluated in MPCs. siRNAs were also assessed for their ability to distinguish between two COL1A1 targets that differ by a single nucleotide $(2 \mathrm{pq}=0.41$, SNP ID: rs $\left.1061237^{7,1,2,4}\right)$. Such sequence specificity would enable generation of two siRNAs targeting each of the polymorphic variants of COL1A1, which together could be used to treat $40 \%$ of OI patients with dominant-negative COL1A1 mutations - those patients who are heterozygous for the polymorphism.

\footnotetext{
Materials and methods siRNAs siRNA target sequences (below) differed by at least four nucleotides from any known gene (BLASTN2.2.6 ${ }^{8}$ ). siRNAs were synthesised $^{5}$ (Qiagen-Xeragon, Germantown, MD 20874, USA). The C/T polymorphism is highlighted in bold print. siRNA7a1, targeting human COL7A1, was used as a negative control.
}

siRNAT1 target: 5' AA CTGAACCCCCTCAAAAGCC 3' (4592-4610 of sense strand Z74615) 
siRNAT1 sense: $\quad 5^{\prime}$ CUGAACCCCCUCAAAAGCC TT 3' siRNAT1 antisense:

3' TT GACUUGGGGGAGUUUUCGG 5'

siRNAT2 target: $5^{\prime}$ CC CCTCAAAAGCCAAAAAATG $3^{\prime}(4600-4618$ of sense strand Z74615)

siRNAT2 sense: $\quad 5^{\prime}$ CCUCAAAAGCCAAAAAAUG GG 3' siRNAT2 antisense:

3' GG GGAGUUUUCGGUUUUUUAC 5'

siRNAC target: $\quad$ 5' CC CCCCAAAAGCCAAAAAATG $3^{\prime}(4600-4618$ of sense strand Z74615)

siRNAC sense: $\quad 5^{\prime}$ CCCCAAAAGCCAAAAAAUG GG 3' siRNAC antisense:

3' GG GGGGUUUUCGGUUUUUUAC 5' siRNA7a1 target: 5' AA GGGGCAGGGGGTCAAGCTA $3^{\prime}(588-516$ of sense strand NM000094)

siRNA7a1 sense: 5' GGGGCAGGGGGUCAAGCUA TT 3' siRNA7a1 antisense:

3' TT CCCCGUCCCCCAGUUCGAU 5'

\section{Target COL1A1 constructs}

siRNA targets (15940-16620 Acc. No. AF017178) with either the T- or C-allele of human COL1A1 were cloned into pIRES2-EGFP (Clontech Laboratories Inc., Palo Alto, USA Cat. No. 6029-1).

Transient transfections of Cos-7 cells and MPCs Cos-7 cells were transfected in quadruplicate in $2 \mathrm{~cm}^{2}$ wells with $0.8 \mu \mathrm{g}$ of target and $50 \mathrm{nmol}$ of siRNA using Lipofectamine 2000. RNA was isolated ${ }^{4}$ after $48 \mathrm{~h}$. MPCs ${ }^{9}$ were transfected with $50 \mathrm{nmol}$ of siRNA using Oligofectamine (Invitrogen Cat. No. 12252-011). RNA was harvested after $7 \mathrm{~h}$. When two siRNAs were cotransfected, $25 \mathrm{nmol}$ of each was used.

\section{Real time RT-PCR}

Quadruplicate transfection samples were pooled and COL1A1 RNA levels analysed by real time RT-PCR, standardised to $\beta$-actin or GAPDH. ${ }^{4} \beta$-Actin expression profile was checked using GAPDH and COL1A2. ${ }^{4}$
ELISA assays

MPC-T/T (below) cells were transfected in quadruplicate with siRNAs. Quadruplicate samples were pooled and ELISAs were performed, in duplicate, $28 \mathrm{~h}$ posttransfection. ${ }^{4}$

\section{Statistical analysis}

ANOVAs and least significant difference tests (LSDs) were performed using Data Desk 6.0 (Data Descriptions Inc., New York, USA). Differences were significant at $P \leqslant 0.05$. Tables include standard errors.

\section{Results \\ siRNA studies for OI therapeutics}

Three mutation-independent siRNAs targeting a polymorphic region in the COL1A1 gene were evaluated in human MPCs. siRNAT1 and siRNAT2 were designed to target the T-allele variant whereas siRNAC targets the C-allele variant of COL1A1..$^{7,1,2,4}$ MPCs were homozygous (MPC-T/T) or heterozygous (MPC-C/T) for the polymorphism.

All siRNAs suppressed COL1A1 (Table 1). siRNAT2 downregulates the T-allele of COL1A1 in MPC-T/T cells more than either siRNAT1 or siRNAC $(84.62,65.23,67.66$ respectively) $(P=0.004$ and 0.01$)$. siRNAT1 and siRNAC are equally active in these cells even though siRNAC has a single-base mismatch with respect to its target $(P=0.7)$. When both siRNAT1 and siRNAC or siRNAT2 and siRNAC are transfected together, lower levels of suppression are achieved than with either siRNAT2 or siRNAC alone, suggesting that the two siRNAs may compete/interfere with one another.

In MPC-C/T cells, siRNAT2 and siRNAC are better than siRNAT1 at suppressing COL1A1 $(P<0.0001)$. Unlike the results found in MPC-T/T cells, siRNAC is better than siRNAT2 at downregulating the combined C- and T-allele transcripts found in the MPC-C/T cells $(P<0.0001)$. This suggests that siRNAC may be better at downregulating the

Table 1 COL1A1 expression levels in MPC-T/T, MPC-C/T, and Cos-7 cells after siRNA transfections

\begin{tabular}{|c|c|c|c|c|}
\hline SiRNA & COL1A1 levels in MPC-T/T (\%) & $n, p$ & COL1A1 levels in MPC-C/T (\%) & $n, p$ \\
\hline $\begin{array}{l}\text { siRNA 7a1 } \\
\text { siRNA T1 } \\
\text { siRNA T2 } \\
\text { siRNA C } \\
\text { siRNA T1+siRNA C } \\
\text { siRNA T2+siRNA C }\end{array}$ & $\begin{array}{r}100 \pm 3.28 \\
34.77 \pm 6.66 \\
15.38 \pm 2.50 \\
32.34 \pm 3.52 \\
45.95 \pm 5.21 \\
46.15 \pm 4.50\end{array}$ & $\begin{array}{c}8 \\
8,<0.0001 \\
8,<0.0001 \\
8,<0.0001 \\
8,<0.0001 \\
8,<0.0001\end{array}$ & $\begin{array}{r}100 \pm 2.67 \\
73.44 \pm 3.40 \\
51.97 \pm 2.46 \\
17.62 \pm 1.24 \\
51.92 \pm 2.64 \\
39.88 \pm 1.14\end{array}$ & $\begin{array}{l}c 16 \\
10,<0.0001 \\
10,<0.0001 \\
8,<0.0001 \\
10,<0.0001 \\
8,<0.0001\end{array}$ \\
\hline SiRNA & T-allele levels in Cos-7 (\%) & $n, p$ & C-allele levels in Cos-7 (\%) & $n, p$ \\
\hline $\begin{array}{l}\text { siRNA 7a1 } \\
\text { siRNA T1 } \\
\text { siRNA T2 } \\
\text { siRNA C }\end{array}$ & $\begin{array}{r}100 \pm 1.46 \\
51.64 \pm 3.36 \\
35.54 \pm 1.97 \\
54.22 \pm 2.55\end{array}$ & $\begin{array}{c}9 \\
9,<0.0001 \\
8,<0.0001 \\
9,<0.0001\end{array}$ & $\begin{array}{r}100 \pm 0.95 \\
65.35 \pm 1.48 \\
38.61 \pm 2.00 \\
47.98 \pm 2.93\end{array}$ & $\begin{array}{cc}9 \\
9,<0.0001 \\
9,<0.0001 \\
9,<0.0001\end{array}$ \\
\hline
\end{tabular}


Table 2 Type I collagen protein expression levels in MPCs after siRNA transfections

\begin{tabular}{lcc}
\hline siRNA or shRNA & $\begin{array}{c}\text { Type I collagen } \\
(\mathrm{ng} / \mathrm{ml})\end{array}$ & $\begin{array}{c}\text { Type I collagen levels in } \\
\text { MPCs (\%) }\end{array}$ \\
\hline siRNA 7a1 & 83.04 & 100 \\
siRNA T1 & 51.48 & 62 \\
siRNA T2 & 23.04 & 28 \\
siRNA C & 43.20 & 52 \\
siRNA T1+siRNA C & 34.08 & 41 \\
siRNA T2+siRNA C & 29.76 & 36 \\
\hline
\end{tabular}

C-allele of COL1A1 than siRNAT2 is at downregulating the T-allele. This is corroborated by the fact that lower levels of suppression are achieved with both siRNAT2 and siRNAC than with siRNAC singly $(P<0.0001)$. When siRNAT1 and siRNAC or siRNAT2 and siRNAC were evaluated in MPCs together, an intermediate level of suppression to that obtained with each siRNA singly was found.

Results quantifying de novo formed Type I collagen protein, excreted from MPC-T/T cells transfected with siRNAs, provide evidence that suppression of RNA is mirrored at the protein level (Table 2). Again, siRNAT2 was the most efficient siRNA, followed by siRNAC and siRNAT1. Protein levels were suppressed by up to $72 \%$.

Since MPC cells with the rare homozygous C-allele genotype were not available, siRNAs were evaluated in Cos-7 cells expressing a partial C- or T-allele COL1A1 gene. Downregulation of up to $65 \%$ was observed. As found in MPCs, siRNAT2 was more active than siRNAT1. In addition, all three siRNAs targeted their own correct target allele better than their incorrect allele, showing allele preference rather than allele-specificity (Table 1).

\section{Discussion}

The use of mutation-independent siRNAs targeting a common and therefore potentially therapeutically significant polymorphism in the COL1A1 gene has been investigated. While levels of COL1A1 suppression of up to $85 \%$ were observed, none of the siRNAs evaluated were found to be completely allele-specific. Specificity of siRNA at the single-nucleotide resolution has been achieved in a number of studies.,10-12 One suggests that to achieve sequence-specificity, small amounts of siRNA should be used. ${ }^{5}$ Another study does not confirm this. ${ }^{5}$ The positioning of a single-nucleotide mismatch within the siRNA molecule at position 9,10 or 11 of the target (from the $5^{\prime}$ end of the target and not including $3^{\prime}$ siRNA overhang sequences) may convey specificity. ${ }^{5,10,11}$ In our study the single mismatch was situated at position 3 (siRNAT2 and siRNAC) or 11 (siRNAT1). Constraints due to COL1A1 target sequence disallowed the study of additional siRNAs targeting this polymorphism.

In summary, RNAi-based suppression of COL1A1 has been demonstrated in MPCs and allele-specificity evaluated. Clearly, in many cases, the lack of allele-specificity will be an enormous barrier when using RNAi to suppress mutant alleles. In addition, constraints incurred by both the target sequence and the necessity to design molecules that will not target multiple genes will be challenging when designing RNAi therapeutics. The use of mutationindependent RNAi targets, such as polymorphic sites, is therefore appealing.

\section{Acknowledgements}

We thank $C$ Allers and $P$ Conget for providing MPCs, and the CBBF, the Health Research Board Ireland and Enterprise Ireland for their support.

\section{References}

1 Millington-Ward S, O’Neill B, Tuohy G et al: Strategems in vitro for gene therapies directed to dominant mutations. Hum. Mol. Genet. 1997; 6: 1415-1426.

2 Millington-Ward S, O'Neill B, Kiang AS, Humphries P, Kenna PF Farrar GJ: A mutation-independent therapeutic strategem for osteogenesis imperfecta. Antisense Nucleic Acid Drug Dev. 1999; 9: 537-542.

3 O'Neill B, Millington-Ward S, O'Reilly M et al: Ribozyme-based therapeutic approaches for autosomal dominant retinitis pigmentosa. Invest. Ophthalmol. Vis. Sci. 2000; 41: 2863-2869.

4 Millington-Ward S, Allers C, Tuohy G et al: Validation in mesenchymal progenitor cells of a mutation-independent ex vivo approach to gene therapy for osteogenesis imperfecta. Hum. Mol. Genet. 2002; 11: 2201-2206.

5 Dykxhoorn DM, Novina CD, Sharp PA: Killing the messenger: short RNAs that silence gene expression. Nat. Rev. Mol. Cell Biol. 2003; 4: 457-467.

6 Horwitz EM, Prockop DJ, Fitzpatrick LA et al: Transplantability and therapeutic effects of bone marrow-derived mesenchymal cells in children with osteogenesis imperfecta. Nat. Med. 1999; 5: 309-313.

7 Westerhausen AI, Constantinou CD, Prockop DJ: A sequence polymorphism in the $3^{\prime}$-nontranslated region of the pro alpha 1 chain of type I procollagen. Nucleic Acids Res. 1990; 18: 4968.

8 Altschul SF, Madden TL, Schaffer et al: Gapped BLAST and PSIBLAST: a new generation of protein database search programs. Nucleic Acids Res. 1997; 25: 3389-3402.

9 Conget PA, Minguell JJ: Phenotypical and functional properties of human bone marrow mesenchymal progenitor cells. J. Cell Physiol. 1999; 181: 67-73.

10 Ding H, Schwarz DS, Keene A et al: Selective silencing by RNAi of a dominant allele that causes amyotrophic lateral sclerosis. Aging Cell 2003; 2: 209-217.

11 Miller VM, Xia H, Marrs GL et al: Allele-specific silencing of dominant disease genes. Proc. Natl. Acad. Sci. USA 2003; 100: $7195-7200$.

12 Abdelgany A, Wood M, Beeson D: Allele-specific silencing of a pathogenic mutant acetylcholine receptor subunit by RNA interference. Hum. Mol. Genet. 2003; 12: 2637-2644. 